ESJ Natural/Life/Medical Sciences

\title{
Effets De Cyperus Articulatus L. (Cyperaceae) Sur La Memoire Des Souris Blanches Suisses (Mus Musculus Swiss.)
}

\author{
Salomon Taah Yamndou,(Msc) \\ Elysée Djedoubouyom Name, (Msc)
}

Centre de Recherche Agricole de Wakwa, Ngaoundéré

Institut de Recherche Agricole pour le Développement (IRAD), Cameroun Asta Madi, (MSc)

Centre de Recherche Agricole de Wakwa, Ngaoundéré

Institut de Recherche Agricole pour le Développement (IRAD), Cameroun

\section{Maxwell Nguedjo Wandji, (Msc)}

Centre de Recherche en Alimentation et Nutrition (CRAN),

Institut de Recherche Médicale et d’Etude des plantes Médicinales,

Cameroun

Francine Mediesse Kengne, (Ph.D)

Centre de Recherche en Plante Médicinale et Médecine Traditionnelle

(CRPMT),

Institut de Recherche Médicale et d’Etude des plantes Médicinales,

Cameroun

Alain Loabe Pahimi, (Ingénieur environnementaliste / Biologiste)

Institut de Recherche Agricole pour le Développement (IRAD), Cameroun

Elisabeth Ngo Bum, (Professeur)

Université de Maroua, Cameroun,

Alexandre Michel Njan Nlôga, (Maître de Conférences)

Université de Ngaoundéré, Cameroun

Doi:10.19044/esj.2021.v17n29p184

Submitted: 11 July 2020

Accepted: 09 September 2020

Published: 31 August 2021
Copyright 2021 Author(s)

Under Creative Commons BY-NC-ND 4.0 OPEN ACCESS

Cite As:

Taah Yamndou S., Djedoubouyom Name E., Asta Madi, Nguedjo Wandji M., Mediesse Kengne F., Loabe Pahimi A., Ngo Bum E. \& Njan Nlôga A.M. (2021). Effets De Cyperus Articulatus L. (Cyperaceae) Sur La Memoire Des Souris Blanches Suisses (Mus Musculus Swiss.). European Scientific Journal, ESJ, 17(29), 184.

https://doi.org/10.19044/esj.2021.v17n29p184 


\section{Résumé}

Le but de la présente étude était d'évaluer l'effet de l'extrait aqueux des rhizomes de Cyperus Articulatus L. (Cyperaceae) sur l'apprentissage et la mémoire des souris blanches Mus musculus Swiss. Les tests du Labyrinthe en $\mathrm{T}$ avec ou sans induction de perte de mémoire ont été réalisé une heure après administration orale de différentes doses d'extrait (52,4, 131, 262 et 524 $\mathrm{mg} / \mathrm{kg}$ ) de C. Articulatus obtenu par décoction. Concernant le test du Labyrinthe en $\mathrm{T}$, l'extrait aux doses tests a entrainé l'augmentation significative de la latence et du nombre d'entrée dans le bras préféré à la phase d'acquisition en comparaison aux souris non traitées. Cependant, seule la dose $131 \mathrm{mg} / \mathrm{kg}$ a significativement réduit le nombre d'entrée dans le bras préféré à la dose $131 \mathrm{mg} / \mathrm{kg}$. Dans le test du labyrinthe en $\mathrm{T}$ avec induction de perte de mémoire, l'extrait à toutes les doses a montré une réduction significative du temps de latence pour entrer dans le bras préféré, du temps passé dans le bras discriminé et du nombre de retour dans le bras de départ. En conclusion, l'extrait aqueux de rhizome de $\mathrm{C}$. Articulatus possède des propriétés qui facilitent la mémorisation et corrigent les déficits de mémoire chez les souris. Les résultats obtenus dans cet article justifient l'utilisation de C. Articulatus en Médecine traditionnelle. Il conviendrait de faire une expérience sur les humains. Le screening phytochimique préliminaire réalisé a montré la présence de flavonoïdes, d'alcaloïdes, de tannins, de saponines, d'anthraquinones, de triterpènes et de polyphénols.

Mots clés: Cyperus Articulatus ; Décoction, Apprentissage, Mémoire, Souris Blanches (Mus musculus Swiss) 


\title{
Effets Of Cyperus Articulatus L. (Cyperaceae) On The Memory Of White Mice (Mus Musculus Swiss)
}

\author{
Salomon Taah Yamndou,(Msc) \\ Elysée Djedoubouyom Name, (Msc)
}

Centre de Recherche Agricole de Wakwa, Ngaoundéré

Institut de Recherche Agricole pour le Développement (IRAD), Cameroun

Asta Madi, (MSc)

Centre de Recherche Agricole de Wakwa, Ngaoundéré

Institut de Recherche Agricole pour le Développement (IRAD), Cameroun

Maxwell Nguedjo Wandji, (Msc)

Centre de Recherche en Alimentation et Nutrition (CRAN),

Institut de Recherche Médicale et d’Etude des plantes Médicinales,

Cameroun

Francine Mediesse Kengne, (Ph.D)

Centre de Recherche en Plante Médicinale et Médecine Traditionnelle

(CRPMT),

Institut de Recherche Médicale et d’Etude des plantes Médicinales, Cameroun

Alain Loabe Pahimi, (Ingénieur environnementaliste / Biologiste)

Institut de Recherche Agricole pour le Développement (IRAD), Cameroun

Elisabeth Ngo Bum, (Professeur)

Université de Maroua, Cameroun,

Alexandre Michel Njan Nlôga, (Maître de Conférences)

Université de Ngaoundéré, Cameroun

\section{Abstract}

The purpose of this study was to evaluate the effect of the water extract of Cyperus Articulatus rhizomes on the learning capacity and memory of Mus musculus Swiss white mice. T-maze tests with or without memory loss induction were performed one hour after oral administration of different doses of extract (52.4, 131, 262 and $524 \mathrm{mg} / \mathrm{kg}$ ) of C. Articulatus obtained by decoction. For the T-maze test, the extract at the various test doses resulted in a significant increase in latency and the number of entries into the preferred arm in the acquisition phase compared to the untreated mice group. However, only the $131 \mathrm{mg} / \mathrm{kg}$ dose significantly reduced the number of entries into the preferred arm. In the T maze test with memory loss induction, the extract at all doses showed a significant reduction in latency to enter the preferred arm, time spent in the discriminated arm and the number of returns to the starting arm. In conclusion, the water extract of $\mathrm{C}$. Articulatus rhizome has properties that facilitate memory and correct memory deficits in mice. Preliminary phytochemical screening showed the presence of flavonoids, alkaloids, 
tannins, saponins, anthraquinones, triterpenes and polyphenols.The results obtained in this article justify the use of C. articulatus in Traditional Medicine. It would be necessary however to conduct similar studies on humans so that these findings can be used to improve memory issues in patients and persons with special needs.

Keywords: Cyperus Articulatus, Decoction, Learning, Memory, Mus musculus Swiss, White Mouse

\section{Introduction}

Les maladies neurodégénératives entraînent la détérioration des cellules nerveuses, affectent le fonctionnement du système nerveux de façon progressive au cours de son évolution. De plus, en fonction des régions atteintes, les troubles pourront affecter la motricité, le langage, la perception, la cognition et la mémoire (Deschamps et Moulignier, 2005). La mémoire qui est la faculté d'enregistrer, de coder, de conserver et de restituer une information en la situant dans le temps est la plus affectée (Atkinson et Shiffrin, 1968). Ces différentes fonctions affectées peuvent modifier l'état de santé d'un individu, sachant que la santé est un état de bien être physique, mental et social (OMS, 2002) qui peut être déséquilibré suite à des perturbations d'origine interne ou externe.

Selon l'OMS, les troubles mentaux et comportementaux représentent $12 \%$ de la charge mondiale de morbidité et si rien n'est fait, cette charge atteindrait les $15 \%$ en 2020 ; ceci entraîne d'importantes conséquences économiques et sociales pour un individu, sa famille et la société. Bien que de nombreuses stratégies de diagnostic et de traitement à base de médicaments conventionnels efficaces aient été établies (quels que soient les traitement mis sur pieds, ces dernièrers sont moins accessibles dans les pays africains en raison des conditions socioculturelles et économiques telles que le côut élevé (OMS, 2001). De plus, ces médicaments présentent des effets secondaires majeurs sur la mémoire (OMS, 2004). Dans le monde, près de $80 \%$ de la population ont recours aux plantes médicinales pour leur problème de santé (OMS, 2008). Cependant, l'adoption de la medécine traditionnelle par les populations pose de nombreux problèmes dus au manque de données scientifiques sur l'innocuité et l'efficacité des préparations traditionnelles consommées. Les propriétés thérapeutiques de certaines plantes restent hypothétiques ou tout simplement, agissent comme des placebos (Ngo Bum, Taiwe, 2004). C'est dans cette optique que les enseignants du Département de Biologie et Physiologie Animales de la Faculté des Sciences de l’Universités de Ngaoundéré ont entrepris de répertorier les plantes médicinales pour déterminer leurs propriétés pharmacologiques, donner des appréciations objectives des propriétés connues, révéler les propriétés inconnues, informer 
la population des méfaits de certaines plantes médicinales et produire des traitements meilleurs sur le marché (Ngo Bum, 2010).

Des études pharmacologiques ont été menées sur Cyperus articulatus (Cypéracéae) dans le but de trouver des molécules actives pour lutter efficacement contre les troubles de la mémoire. Des études ont révelé que la décoction de cette plante est utilisée dans le traitement de plusieurs pathologies telles que la toux, la fièvre, la coqueluche (Walker et Silans, 1961 ; Vergiat, 1970 ; Schultes et Raffaud, 1990), les bactéries (Duarte, 2005). Elle a également fait ses preuves dans le traitement de certaines maladies du système nerveux central telles que l'épilepsie (Nkantchoua, 2004 et Ngo Bum, 2010), l'insomnie (Rakotonirina, 2001) et la dépression. Cependant, aucune recherche n'a jamais été faite sur la mémoire.

Ainsi, il est principalement question de mettre en évidence des effets de la décoction des rhizomes de Cyperus Articulatus sur les capacités mnésiques chez les souris blanches Suisses Mus musculus L. (Muridae).

\section{Matériel et Méthodes}

\section{Préparation de la décoction des rhizomes de Cyperus Articulatus}

Les racines de rhizones collectées dans une banlieu de Yaoundé, ont été séchées à l'ombre pendant 2 semaines et ensuite pilés dans un mortier en bois. La poudre de rhizomes (2,5 g) de C. articulatus obtenue est introduite dans un bécher contenant $25 \mathrm{~mL}$ d'eau distillée. Le mélange a été macéré pendant $18 \mathrm{~h}$ puis porté à ébullition pendant 20 min sur une plaque chauffante réglée à $100{ }^{\circ} \mathrm{C}$. Après refroidissement le mélange a été filtré à l'aide d'un papier filtre Wattman $\mathrm{n}^{\circ} 4$ et le filtrat récupéré constituait la solution mère. $\mathrm{A}$ partir de la solution mère, des dilutions au 1/2, 1/4,1/8, 1/10 ont été réalisées pour obtenir les différentes doses test.

Le filtrat (solution mère) dont le volume mesuré $21 \mathrm{~mL}$ a été récupéré et porté à évaporation à l'étuve $\left(80^{\circ} \mathrm{C}\right)$ pendant $24 \mathrm{~h}$. Nous avons obtenu 1,31 g d'extrait sec de $C$. articulatus soit un rendement de 0,524 \% dont la concentration est $100 \mathrm{mg} / \mathrm{mL}$. Le volume d'administration étant de $10 \mathrm{~mL} / \mathrm{kg}$, les différentes doses test étaient de 524; 262; 131 et 52,4 mg/kg.

\section{Tests de caractérisation phytochimique}

Les tests préliminaires de caractérisation phytochimique de la décoction de C. Articulatus, ont été réalisés par des méthodes qualitatives colorimétriques (Harbone, 1973), pour la détermination des principaux groupes chimiques.

1. Test des alcaloïdes. Un mililitre de décoction des rhizomes de $C$. Articulatus a été mélangé à quelques gouttes d'acide sulfurique (2\%) et quelques gouttes de réactif de Meyer. La formation d'un précipité blanc indiquait la présence d'alcaloïdes dans le milieu. 
2. Test des saponines. Un mililitre de décoction a été vigoureusement agité. Après agitation, la formation d'une mousse persistante après une durée de 15 min rendait compte de la présence de saponine dans le milieu.

3. Test des flavonoïdes. Deux mililitres de la décoction de $C$. Articulatus ont été mélangés à $2 \mathrm{~mL}$ de soude à $1 \mathrm{~N}$. La présence d'une coloration jaune qui disparait aussitôt après addition de l'acide chlorhydrique a confirmé la présence des flavonoïdes dans le milieu.

4. Test des tanins. Deux mililitres de la décoction de $C$. Articulatus ont été mélangés à $2 \mathrm{~mL}$ de sulfate de cuivre. Au mélange, est ajouté 2 gouttes d'ammoniaque. La formation d'un précipité noir, bleu ou vert indique la présence de tanins dans le milieu.

5. Test des triterpènes. Trois mililitres de la décoction sont mélangés à quelques gouttes d'anhydride acétique puis à ajouter quelques gouttes d'acide sulfurique. La formation d'une coloration rouge violacée indique la présence des triterpènes dans le milieu.

6. Test des anthraquinones. Un mililitre de la décoction est mélangé au $\mathrm{NaOH} 50 \%$, et nous avons le développement d'une coloration rouge ou violette indiquant la présence effective des anthraquinones dans notre extrait.

\section{Tests pharmacologiques}

\section{Préparation des animaux}

Les souris blanches Mus musculus Swiss, naïves, adultes, des deux sexes, pesant entre 18 et 30 g ont été utilisées pour réaliser les différents tests. Les animaux ont été acclimatés pendant 72 heures à la température ambiante $\left(20 \pm 1^{\circ} \mathrm{C}\right)$ du laboratoire avant le début des manipulations et étaient nourris matin et soir à base de granulés. Ces animaux étaient soumis à un cycle de 12 heures de lumière et 12 heures d'obscurité. Les différents tests ont été effectués entre 9 heures et 15 heures.

\section{Effet de la décoction de Cyperus articulatus sur la mémoire des souris dans le labyrinthe en $T$}

Les effets de C. Articulatus sur le niveau d'exploration, l'apprentissage et la mémoire chez la souris naïve (souris non manipulée) placée dans le labyrinthe en T ont été évalués. Deux jours avant le début des expériences, les animaux sont progressivement privés de nourriture pour les maintenir à 80-85 $\%$ de leur poids. Ces derniers sont réparties en 6 lots homogènes de 5 animaux chacun. Ces animaux ont reçu de l'eau distillée pour le lot contrôle négatif, le diclofénac $(5 \mathrm{mg} / \mathrm{kg}$ ) pour le lot contrôle positif et les différentes doses de la décoction de $C$. Articulatus pour les lots tests. Les souris sont placées les unes après les autres dans le bras de départ du labyrinthe en $\mathrm{T}$ une heure après l'administration des différentes substances. Cette tâche s'est déroulée en trois phases : l’habituation, l'acquisition et la rétention. 
Dans la première phase ou phase d'habitation, les souris sont familiarisées avec l'appareil pendant une période de 5 minutes. De la nourriture est placée dans chacun des couloirs pour favoriser l'exploration. L'expérimentateur met la souris dans le compartiment de départ. Après $15 \mathrm{sec}$, il ouvre toutes les portes à guillotines. L'animal peut alors choisir l'un ou l'autre couloir des bras de l'appareil indiquant ainsi sa préférence. Les paramètres enregistrés étaient les suivants : le temps de latence pour choisir un bras (le bras qui constituera le bras préféré de l'animal tout au long du test), le temps passé et le nombre d'entrées dans le bras préféré et le bras discriminé, et le nombre de retour dans le bras de départ.

La deuxième phase ou phase d'acquisition, commence 24 heures après la phase d'habituation. Le couloir du bras discriminé par l'animal est fermé, puis un renforçateur (nourriture) est placé dans le bras choisi par l'animal. L'expérimentateur met la souris dans le compartiment de départ et la laisse se déplacer vers le couloir ouvert (couloir préféré pendant l'habituation). Cette phase s'effectue en 5 min pour chaque animal. Les paramètres suivants sont notés: le temps de latence pour retrouver la nourriture, le temps passé et le nombre d'entrées dans les bras préférés le nombre de retour dans le bras de départ.

Enfin vient la phase de rétention 24 heures après la phase d'acquisition. Chaque animal est placé pour une durée de 5 min dans l'appareil, cette fois-ci tous les bras du dispositif sont ouverts. L'expérimentateur place la nourriture dans les deux bras du labyrinthe. Les paramètres enregistrés sont : le temps de latence pour retrouver le bras préféré, le temps passé et le nombre d'entrées dans le bras préféré et le bras discriminé, et le nombre de retour dans le bras de départ. Après les 5 min d'expérimentation

\section{Effet de la décoction de Cyperus Articulatus sur la mémoire des souris ayant reçu la scopolamine dans le Labyrinthe en $T$}

Les effets de $C$. Articulatus sur la perte de mémoire induites par la scopolamine ont été évalués chez la souris naïve placée dans le labyrinthe en $\mathrm{T}$, les animaux sont répartis en 6 lots homogènes de 5 souris. Ils sont répartis comme suit : 1 lot contrôle négatif qui a reçu seulement de l'eau distillée (10 $\mathrm{mg} / \mathrm{kg}), 1$ lot contrôle scopolamine qui a reçu de l'eau distillée $(10 \mathrm{mg} / \mathrm{kg})$ et de la scopolamine $(0,1 \mathrm{mg} / \mathrm{kg})$ une heure plus tard, 1 lot contrôle positif qui a reçu de la scopolamine $(0,1 \mathrm{mg} / \mathrm{kg})$ une heure plus tard, les lots tests qui ont reçu les différentes doses de la décoction de de C. articulatus (52.4, 131, $262,524 \mathrm{mg} / \mathrm{kg})$ et de la scopolamine $(0,1 \mathrm{mg} / \mathrm{kg})$ une heure plus tard. Toutefois, ces administrations n'ont eu lieu que pendant la phase de rétention. Pendant la phase d'habitation et d'acquisition les animaux ont été soumis à l'entrainement. Les souris sont placées les unes après les autres dans le bras de départ du labyrinthe en $\mathrm{T}, 30$ minutes après l'administration de la 
scopolamine pour les animaux du contrôle positif et les lots tests et 30 minutes après l'administration de l'eau distillée pour les animaux du contrôle négatif.

Les mêmes paramètres sont enregistrés comme dans le labyrinthe $\mathrm{T}$ normale pour la phase d'habitation et d'acquisition. Quant à la phase de rétention, le pourcentage de réussite n’est pas enregistré.

\section{Analyse des Résultats}

Un test de normalité et d'homoscédasticité à été effecctué. Les données ont été exprimées en moyennes \pm écart-types. L'analyse de la variance (ANOVA) a été utilisée pour la comparaison des moyennes suivie du test de comparaison des variables de Dunnett et Turkey effectué à l'aide du logiciel XL.Stat version 2007. Les différences ont été considérées comme significatives à partir de $\mathrm{p} \leq 0,05$.

\section{Résultats}

\section{Phase d'habituation.}

\section{Effets sur le temps de latence pour entrer dans le bras d'arrivée}

Une réduction de temps de latence par des souris traitées par les différentes doses de la décoction de $C$. Articulatus par rapport à celles du lot témoin négatif est observée (Figure 1). Le temps de latence pour trouver le bras d'arrivée pour la première fois qui passe de 22,6 $\pm 5,1$ secondes chez les souris du lot témoin négatif à 10,6 \pm 2,7 secondes chez celles traitées par la dose $131 \mathrm{mg} / \mathrm{kg}$ de C. Articulatus.

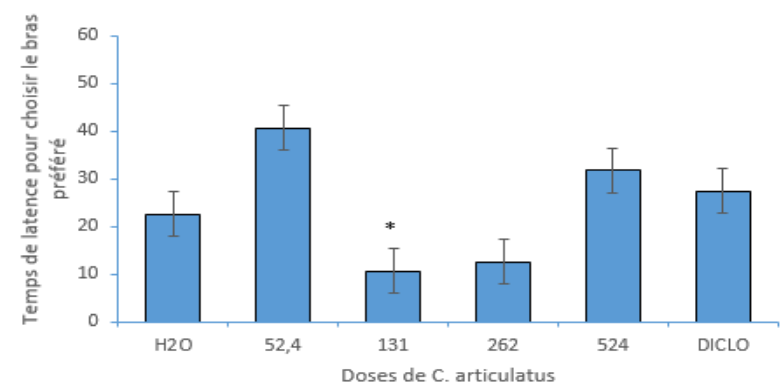

Figure 1. Effet de la décoction de Cyperus Articulatus sur le temps de latence.

Chaque barre représente la moyenne \pm écart type ; $n=5$. H20: Eau distillée (contrôle négatif); DICLO $=$ diclofenac (contrôle positif). ${ }^{*} \mathrm{p} \leq 0,05 ; * * * \mathrm{p} \leq 0,001$ différence significative par rapport au contrôle négatif

\section{Effet de $\boldsymbol{C}$. Articulatus sur le nombre de retour dans le bras de départ}

Une réduction significative du nombre de retour dans le bras de départ est également notée à la Figure 2 ci-contre. Ce nombre qui passe de 6,4 $\pm 2,0$ secondes pour le control négatif à $1,6 \pm 0,5$ seconde pour les souris traitées par la dose $262 \mathrm{mg} / \mathrm{kg}$; et 2,8 \pm 1,4 secondes pour le diclofénac qui induit aussi 
une diminution significative par rapport au lot témoin qui est de $6,4 \pm 2,0$ secondes.

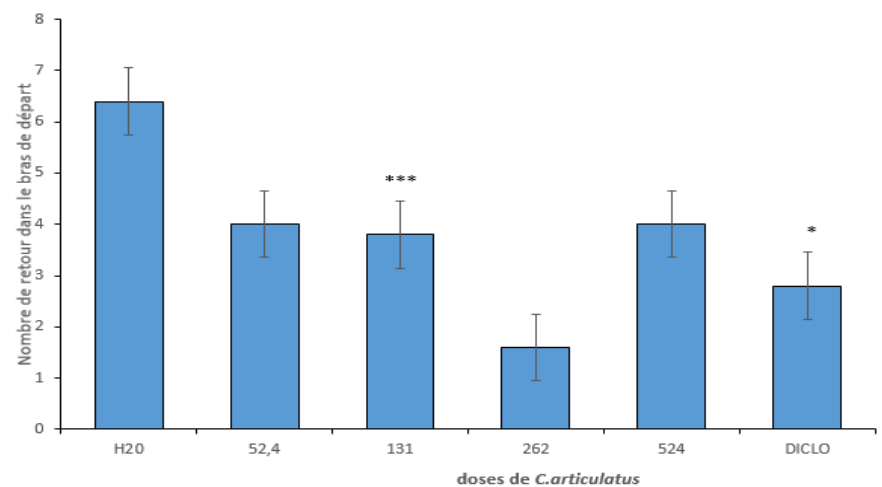

Figure 2. Effet de la décoction de Cyperus Articulatus sur le nombre de retour dans le bras de départ

Chaque barre représente la moyenne $\pm \mathrm{ESM} ; \mathrm{n}=5 .{ }^{*} \mathrm{p} \leq 0,05,{ }^{* * *} \mathrm{p}$ $\leq 0,001$ différence significative par rapport au contrôle négatif. ED $=$ eau distillée, Diclo = diclofénac $5 \mathrm{mg} / \mathrm{kg}$ (contrôle positif).

Effet de la décoction de $C$. articulatus sur le temps passé dans le bras préféré du labyrinthe en $\mathbf{T}$

La décoction de $C$. Articulatus provoque une augmentation significative sur le temps passé dans le bras préféré. Ce nombre passe de 153,8 $\pm 30,2$ secondes pour le lot témoin négatif à $217 \pm 36$ secondes pour la dose $524 \mathrm{mg} / \mathrm{kg}$ et $233 \pm 27$ secondes pour le diclofénac $5 \mathrm{mg} / \mathrm{kg}$.

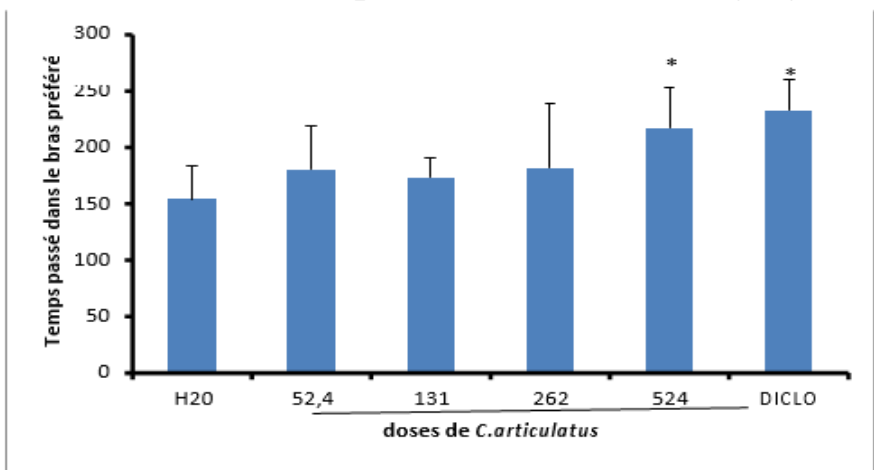

Figure 3. Effet de Cyperus Articulatus sur le temps passé dans le bras préféré

Effet de la décoction de Cyperus Articulatus sur le nombre d'entré dans le bras préféré du labyrinthe en $T$

Une augmentation significative du nombre d'entré dans le bras préféré du labyrinthe est notée (Figure 4). Ce nombre qui passe de $2 \pm 0,7$ secondes 
pour le control négatif à 2,6 \pm 0 secondes et 4,8 $\pm 0,8$ secondes pour les doses 262 et $524 \mathrm{mg} / \mathrm{kg}$ respectivement de la décoction de C. Articulatus.

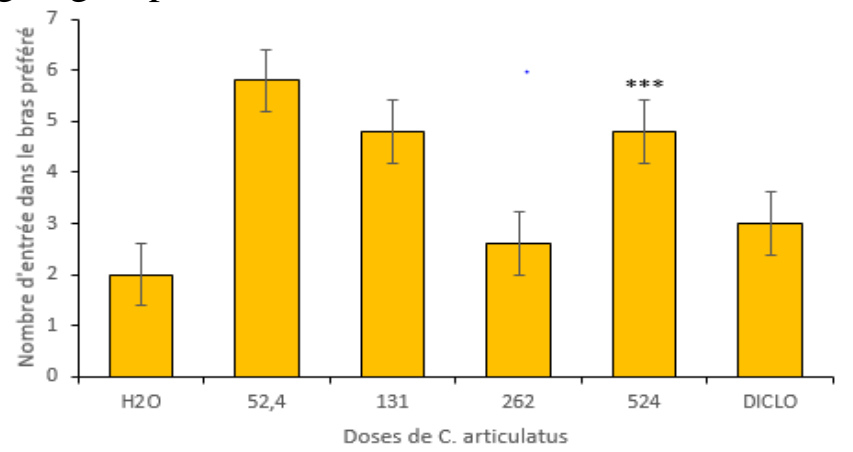

Figure 4. Effet de la décoction de Cyperus Articulatus sur le nombre d'entrée dans le bras préféré du labyrinthe en $\mathrm{T}$

Effet de la décoction de $C$. Articulatus sur le nombre de retour dans le bras de départ

Le nombre de retour dans le bras de départ diminue significativement de 6,2 $\pm 0,8$ secondes pour le lot négatif à 5,4 et à 5,6 \pm 0,5 secondes pour les doses respectives 52,4 et $131 \mathrm{mg} / \mathrm{kg}$ et $4 \pm 0,7$ secondes pour le diclofénac qui induit une diminution significative par rapport au lot négatif (Figure 5).

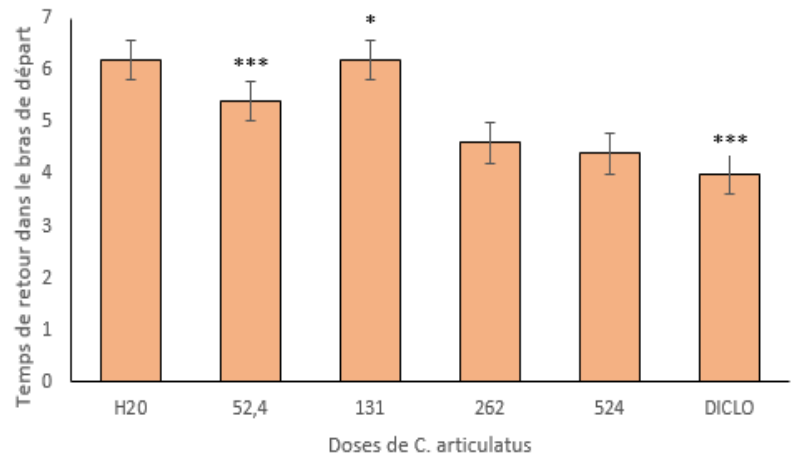

Figure 5. Effet de Cyperus Articulatus sur le nombre retour dans le bras de départ dans le labyrinthe en $\mathrm{T}$

\section{Phase de rétention}

Effet de Cyperus Articulatus sur le temps de latence du premier choix du bras d'arrivée

On note ici une diminution du temps de latence du premier choix du bras d'arrivée. Ce temps qui va de 51,4 $\pm 3,36$ secondes pour le controle négatif à 20,6 \pm 5,50 secondes pour la dose $131 \mathrm{mg} / \mathrm{kg}$. 


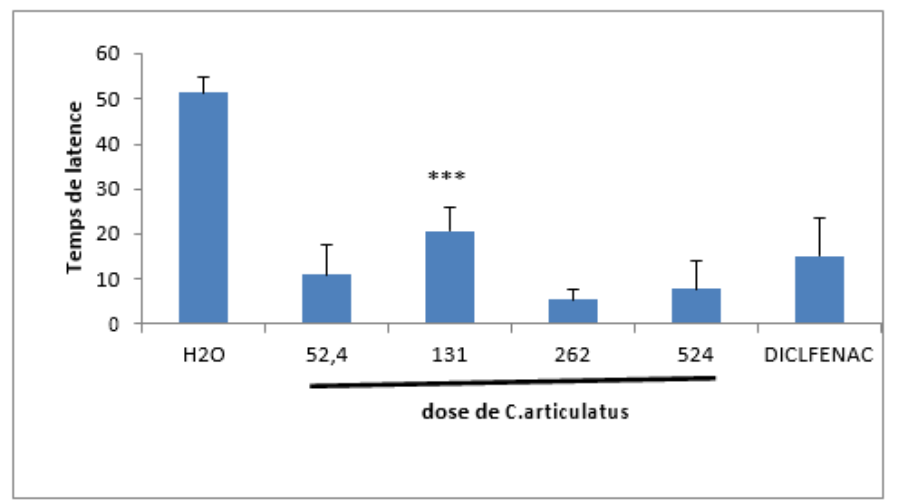

Figure 6. Effet de la décoction de Cyperus Articulatus sur le temps de latence du choix du bras d'arrivée

Effet de la décoction de Cyperus Articulatus sur le temps passé dans les bras d'arrivée (préféré et discriminé) dans le labyrinthe en $T$

Le temps d'entré dans le bras discriminé par rapport au control négatif diminue significativement (Figure 7). Ce temps passe pour le contrôle négatif à $120 \pm 20$ secondes et 128,25 secondes pour les doses 131 et $524 \mathrm{mg} / \mathrm{kg}$ et augmente de 108,8 $\pm 22,0$ secondes pour le control négatif à 135,4 $\pm 35,3$ secondes et pour le diclofénac $5 \mathrm{mg} / \mathrm{kg}$.

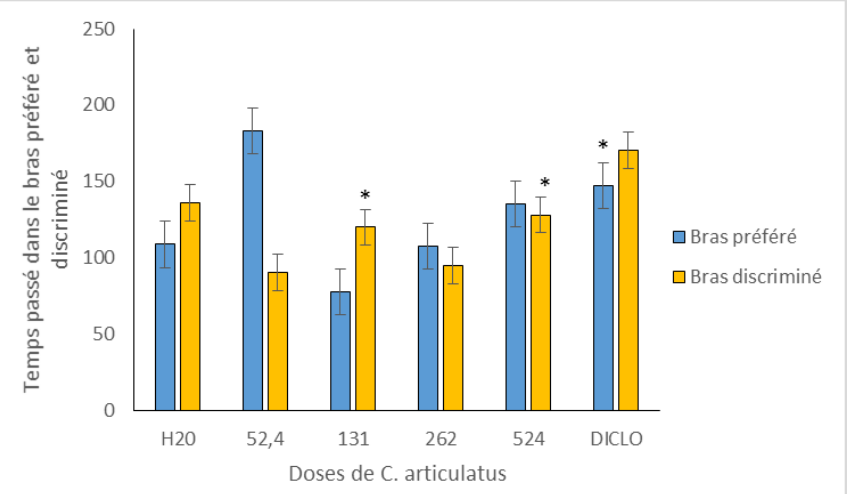

Figure 7. Effet de la décoction de Cyperus Articulatus sur le temps passé dans le bras préféré et discriminé du labyrinthe en $\mathrm{T}$

Chaque barre représente la moyenne $\pm \mathrm{ESM} ; \mathrm{n}=5 . * \mathrm{p} \leq 0,05, * * * \mathrm{p}$ $\leq 0,001$ différence significative par rapport au contrôle négatif. ED = eau distillée, Diclo = diclofénac $5 \mathrm{mg} / \mathrm{kg}$ (contrôle positif). 
Effet de la décoction de Cyperus Articulatus sur la mémoire avec induction de la scopolamine dans le test du labyrinthe en $T$ pendant la rétention Effet de la décoction de Cyperus Articulatus sur le temps de latence du bras préféré du labyrinthe en $T$

Le temps de latence du bras préféré pendant la rétention est réduit significativement (Figure 8). Ce temps passe de 26,8 \pm 3,9 secondes pour le contrôle négatif scopolamine à 17,4 $\pm 3,2$ seconde pour la dose $524 \mathrm{mg} / \mathrm{kg}$.

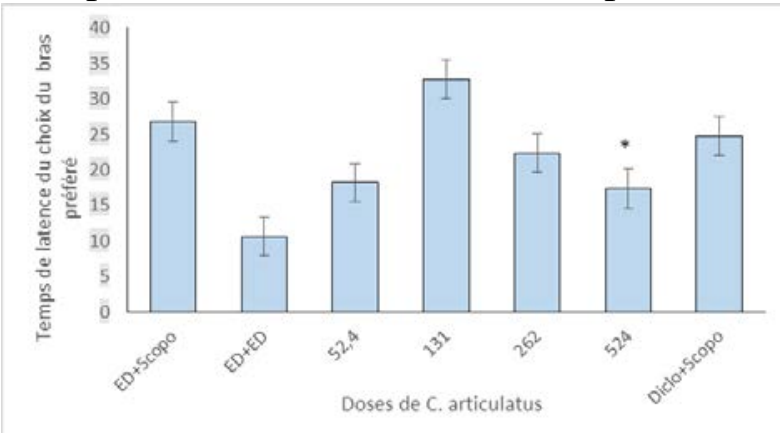

Figure 8. Effet de la décoction de Cyperus Articulatus sur le temps de latence induit par scopolamine pendant la rétention

Chaque barre représente la moyenne $\pm \mathrm{ESM} ; \mathrm{n}=5 .{ }^{*} \mathrm{p} \leq 0,05$, différence significative par rapport au contrôle négatif. $\mathrm{ED}=$ eau distillée, Diclo = diclofénac $5 \mathrm{mg} / \mathrm{kg}$ (contrôle positif), scopo = scopolamine 0,3 mg/kg (ANOVA suivie du test de comparaison multiple de Dunnett).

Effet de Cyperus Articulatus sur le nombre de retour dans le bras de départ pendant la phase de rétention

La décoction du rhizome de $C$. Articulatus ne montre aucun effet (il n'est tout de même pas nul) pour toutes les doses sur le nombre de retour dans le bras de départ.

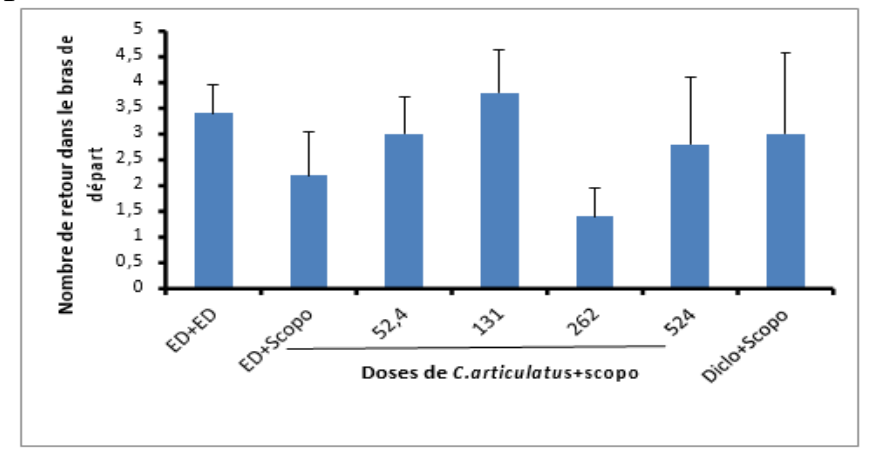

Figure 9. Effet de Cyperus Articulatus sur le nombre de retour dans le bras de départ pendant la phase de retention. 


\section{Effet Cyperus Articulatus sur le temps passé dans les bras d'arrivée (bras} préféré et discriminé) du labyrinthe en $T$ pendant la phase de rétention

Une augmentation et une diminution significatives de temps passé dans les bras d'arrivée (préféré et discriminé) par les souris traitées par les différentes doses de $C$. Articulatus sont notées. Cette augmentation qui passe de 39,4 $\pm 3,9$ secondes pour le lot témoin négatif à 120,8 \pm 29 ,8 secondes et $137 \pm 20,7$ secondes; et 114,6 \pm 11 secondes pour les doses 52,4; 262; et 524 $\mathrm{mg} / \mathrm{kg}$ (Figure 10). Pourtant, ces mêmes doses induisent une diminution significativement du temps passé dans le bras discriminé, y compris le contrôle positif.

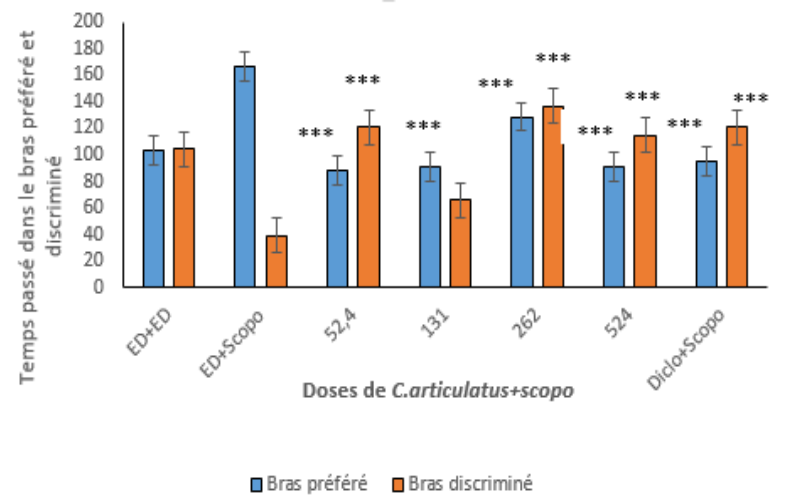

Figure 10. Effet de la décoction de Cyperus Articulatus sur le temps passé dans bras d'arrivée (préféré et discriminé) du labyrinthe en T

Chaque barre représente la moyenne $\pm \mathrm{ESM} ;{ }^{* * *} \mathrm{p} \leq 0,001$ différence significative par rapport au contrôle négatif scopolamine (ED + Scopo). ED = eau distillée, Scopo = scopolamine 0,3 mg/kg, Diclo = diclofénac $5 \mathrm{mg} / \mathrm{kg}$ (contrôle positif).

Effet de Cyperus Articulatus sur le nombre d'entrées dans les bras d'arrivée (bras préféré et discriminé) du labyrinthe en $T$ pendant la phase de la rétention

La scopolamine diminue le nombre d'entrées dans le bras préféré et la décoction de $C$. Articulatus lève cette diminution en augmentant significativement ce nombre qui passe de 1,8 $\pm 0,85$ secondes (bras préféré) chez les souris du lot témoin scopolamine à $4,8 \pm 1,9$ secondes pour la dose $262 \mathrm{mg} / \mathrm{kg}$; pourtant le diclofenac $5 \mathrm{mg} / \mathrm{kg}$ induit une augmentation significative qui est de $5 \pm 1,5$ secondes et toute les autres doses de décoction y compris le contrôle positif dans le bras discriminé sont sans effet (Figure 11). 


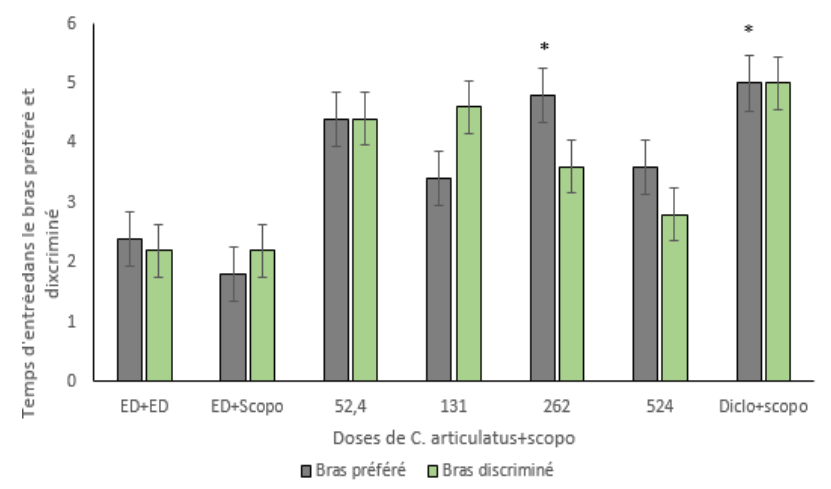

Figure 11. Effet de la décoction de Cyperus Articulatus sur le nombre d'entrées dans les bras d'arrivées (préféré et discriminé)

Chaque barre représente la moyenne $\pm \mathrm{ESM} ; \mathrm{n}=5 .{ }^{*} \mathrm{p} \leq 0,05$ différence significative par rapport au contrôle négatif scopolamine (ED + Scopo). ED = eau distillée, Scoposcopolamine 0,3 mg/kg, Diclo = diclofénac $5 \mathrm{mg} / \mathrm{kg}$ (contrôle positif).

\section{Discussion}

Les résultats obtenus lors du test du labyrinthe en $\mathrm{T}$ ont montré une diminution à la fois du temps de latence, du temps passé dans le bras discriminé, du nombre de retour dans le bras de départ d'une part, d'autre part une augmentation du nombre d'entré et du temps passé dans le bras préféré, du nombre d'entré dans le bras discriminé chez les souris ayant reçu la décoction de $C$. Articulatus. Cette diminution du temps de latence pour retrouver la nourriture suggère une amélioration de la mémoire de référence. En même temps l'augmentation du nombre d'entrées dans les bras d'arrivées montre qu'il y a eu augmentation de l'exploration. Par ailleurs, l'amélioration des capacités mnésiques et la réduction de stress par la présence des renforçateurs alimentaires sont favorables pour un bon fonctionnement de la mémoire. Des résultats similaires ont été obtenus par Farshchi et Ghiasi (2010) ; Heru et al. (2017). Des études ont montré que le stress peut détériorer ou améliorer la mémoire selon Song Li et Ya-Xin F. (2012). On peut donc dire que la décoction du rhizome de $C$. Articulatus aurait des propriétés qui facilitent l'apprentissage et la mémorisation après administration au cours des différentes phases du test.

Pour confirmer ces effets positifs de la décoction de $C$. Articulatus sur la mémoire, le test d'induction de la perte de mémoire par la scopolamine (antagonisme muscarinique ) a été utilisé. La diminution remarquable du nombre de retour dans le bras du départ, du temps passé dans les bras et du temps de latence pour retrouver la nourriture chez les souris par rapport à celles qui ont reçu l'eau distillée et la décoction des différentes doses de C. 
Articulatus, montrent que C. Articulatus antagoniserait l'effet de la scopolamine et aurait une action sur l'amélioration de la mémoire. De même, l'augmentation du nombre d'entré et du temps passé dans les bras d'arrivées suppose un accroissement de l'exploration dans le test du labyrinthe en $\mathrm{T}$ chez les souris ayant reçu les différentes doses de C. Articulatus.

\section{Conclusion}

La présente étude menée sur la décoction des rhizomes de Cyperus Articulatus nous a permis de mettre en évidence ses effets sur le système nerveux central précisément sur la mémoire. Les effets de la décoction de $C$. Articulatus entraînent une augmentation des capacités mnésiques, ainsi que des composés antagonistes de la scopolamine (antagoniste des récepteurs muscariniques). L'ensemble de ces observations laisse penser que $C$. Articulatus possède des propriétés améliorant les capacités mnésiques. Propriétés qui seraient dues à la présence dans la décoction des flavonoïdes, des alcaloïdes, des saponines, des tanins, tri terpènes, poly-phénols dont les tanins et flavonoïdes et des anthraquinones qui agiraient par fixation sur certains récepteurs du système nerveux central. Tous ces effets nous permettent de comprendre au moins en partie l'utilisation des rhizomes de $C$. Articulatus en médecine traditionnelle. Bien que d'autres investigations soient nécessaires, ces résultats montrent que la décoction de $C$. Articulatus peut être utilisée comme traitement des atteintes de la mémoire comme en médecine traditionnelle.

\section{Recommendations}

Pour les analyses ultérieures, les chercheurs recommendent:

- D’utiliser différents agonistes et antagonistes des récepteurs impliqués dans les atteintes de la mémoire pour mettre en évidence le mécanisme d'action des composés contenus dans la décoction de Cyperus Articulatus;

- D'étudier et quantifier les familles chimiques présentes dans la décoction de Cyperus Articulatus ;

- D'étudier la toxicité aigüe et chronique et subchronique de la décoction de C. Articulatus.

\section{References:}

1. Atkinson R. \& Shiffrin R. (1968). Human memory: A proposed system and its control processes. K W Spence, J T Spence Edition Psychology of Learning and Motivation: Advances in Research and Theory., 2, 89195 doi: 10.10/6/S0079-7421(08)60422-3.

2. Deschamps R. \& Moulignier A. (2005). La mémoire et ses troubles. Memory and related disorders.EMC-Neurologie2. J. Neuroscience., 2: 505-525, doi: 10.1016/j.emcn.2005.07.003. 
3. Duarte M. (2005). Anti-Candida activity of Brazilian medicinal plants. J. Ethnopharmacol., 97: 305-311, doi : 10.1016/j.jep.2004.11.016.

4. Farshchi A., Ghiasi G., Farshchi S. \& Khatabi M. (2010). Effects of Boswellia Papyrifera Gum Extract on Learning and Memory in Mice and Rats. Iran. J. Basic. Med. Sci. ; 13(2), 9-15.

5. Taiwe G., Ngo Bum E. \& Théophile, Dima T., Weiss N. (2010). Antidepressant, myorelaxant and anti-aniety-like effects of Nauclea latifolia smith (Rubiaceae) roots extract in murine models. Int. J. Pharmacolocy., 6 (4), 364-371, doi : 10.3923/ijp.2010.364.371.

6. Harborne J.B. (1973). Phytochemical Methods: A guide to modern techniques of plant analysis. Chapman \& Hall, London. D'action des extraits des rhizomes de Cyperus articulatus (cypéracée). Thèse doctorat, en neuropharmacologie. Université de Yaoundé1.Cameroun, $278 \mathrm{p}$.

7. Heru S. (2017). The effect of Cyperus Rotundus on Memory and the Numbe of Hippocampus Cells on Alcohol Administration. ARC Journal of Nursing and Healthcare, 3(2) : 15-17.

8. Ngo Bum E. (2004). Détermination des effects anticonvulsants et certains mécanismes d'action des extraits des rhizomes de Cyperus articulatus (cypéracée). Thèse Doctorat, en neuropharmacologie. Université de Yaoundé1.Cameroun.

9. Ngo Bum E., Nkantchoua, G.- N., Njifutie Njikam., Taiw, G.- S., Ngoupaye G.- T., Pelanken M.- M., Manga L., Maidawa F., Rakotonirina A., Rakotonirina S.- V. (2010). Anticonvulsant and sedative activiçty of leaves of Senna spectzbilis in mice. Int. J. Pharmacol, (6) :123-128, doi : 10.3923/ijp.2010.123.128.

10. OMS. ( 2001a). Communiqué de presse numéro 18. Genève: Organisation Mondiale de la Santé.

11. OMS. (2001b). Communiqué de presse numéro 18. Genève: Organisation Mondiale de la Santé.

12. OMS. (2001). Communiqué de presse numéro 18. Genève: Organisation Mondiale de la Santé.

13. OMS. (2002). Médecine traditionnelle : besoins croissants et potentiels. Bulletin Organisation Mondiale de la Santé.

14. OMS. (2004). Médecine traditionnelle: besoins croissants et potentiels. Bulletin

15. Organisation Mondiale de la Santé.

16. OMS. (2008).Stratégie de l’OMS sur la médecine Traditionnelle; Organisation Mondiale de la Santé; Genève.

17. Rakotonirina S.-V., Ngo Bum E., Rakotonirina A. \& Bopelet M. (2001). Sedatives properties of the total extract of the rhizomes of 
Cyperus articulates. L. Fitoterapia, 72 (1): 22-29, doi: 10.1016/S0367326X (00)00243-4.

18. Schultes, R.-E \& Raffaud R.-F. (1990). The hearling forest: Medicinal plants of the Northwest Amazonia. Dioscorides Press, Portland, p.156, ISBN13: 9780931146145.

19. Song Li, Ya-Xin F., Wei W. \& Yi-Yuan T. (2012). Effects of acute restraint stress on different components of memory as assessed by object-recognition and object- location tasks in mice. Behavioural BrainResearch. 227 (1): 199-207, doi: 1016/j.bbr.2011.10.007.

20. Vergiat A.- M. (1970). Plantes magiques et médécinales des féticheurs de l’Oubangui (Région de Bangui). J. Agri. Trop. Bot. Appli, 17 (5-6): 295-339, doi: 10/3406/jatba.1970.3071.

21. Walker A. R. \& Silans R. (1961). Les plantes Utiles du Gabon. Paul Lechevalier, Paris. pp : 19-132, ISBN-13 : 978-2907888721. 